University of Windsor

Scholarship at UWindsor

1983

\title{
1/Z expansion calculation of the Bethe logarithm for the ground state Lamb shift of two-electron ions
}

S. P. Goldman

Gordon W. F. Drake

University of Windsor

Follow this and additional works at: https://scholar.uwindsor.ca/physicspub

Part of the Physics Commons

\section{Recommended Citation}

Goldman, S. P. and Drake, Gordon W. F.. (1983). 1/Z expansion calculation of the Bethe logarithm for the ground state Lamb shift of two-electron ions. Journal of Physics B: Atomic and Molecular Physics, 16 (7), L183-L187.

https://scholar.uwindsor.ca/physicspub/39

This Article is brought to you for free and open access by the Department of Physics at Scholarship at UWindsor. It has been accepted for inclusion in Physics Publications by an authorized administrator of Scholarship at UWindsor. For more information, please contact scholarship@uwindsor.ca. 
$1 / Z$ expansion calculation of the Bethe logarithm for the ground state Lamb shift of twoelectron ions

This article has been downloaded from IOPscience. Please scroll down to see the full text article.

1983 J. Phys. B: At. Mol. Phys. 16 L183

(http://iopscience.iop.org/0022-3700/16/7/001)

View the table of contents for this issue, or go to the journal homepage for more

Download details:

IP Address: 137.207.184.30

The article was downloaded on 26/04/2013 at $15: 42$

Please note that terms and conditions apply. 


\title{
LETTER TO THE EDITOR
}

\section{$1 / Z$ expansion calculation of the Bethe logarithm for the ground state Lamb shift of two-electron ions}

\author{
S P Goldman $\dagger$ and G W F Drake $\ddagger \S$ \\ + Department of Physics, University of Western Ontario, London, Ontario, Canada \\ $\ddagger$ Research and Engineering Staff, Ford Motor Company, Dearborn, Michigan 48121, USA
}

Received 22 December 1982

\begin{abstract}
The leading two terms in the $1 / Z$ expansion of the two-electron Bethe logarithm are calculated by the application of a new finite basis set method. The results can be expressed in the form

$$
\ln \varepsilon\left(1 \mathrm{~s}^{2} \mathrm{~S} \mathrm{~S}\right)=\ln \left[19.77(Z-0.0063)^{2}\right] \text {. }
$$

The high- $Z$ behaviour appears to differ from that of a previous variational calculation by Aashamar and Austvik.
\end{abstract}

Recent measurements of transition frequencies in high- $Z$ two-electron ions (Davis and Marrus 1977, Berry et al 1978, 1980, O'Brien et al 1979, Armour et al 1979, Holt et al 1980, DeSerio et al 1981, Buchet et al 1981, Stamp et al 1981) are sufficiently accurate to be sensitive to the lowest-order Lamb shift type radiative corrections. The principal uncertainty in the theoretical calculation of the Lamb shift is the value of the two-electron Bethe logarithm defined by

$$
\ln \varepsilon(n L S)=\frac{\Sigma_{m}\left|\left\langle\psi_{0}|t| \psi_{m}\right\rangle\right|^{2} \ln \left(E_{m}-E_{0}\right) /\left(E_{m}-E_{0}\right)}{\Sigma_{m}\left|\left\langle\psi_{0}|t| \psi_{m}\right\rangle\right|^{2} /\left(E_{m}-E_{0}\right)}
$$

in the dipole acceleration form, where $\psi_{0}\left(E_{0}\right)$ is the wavefunction (energy) for the $n L S$ two-electron configuration, $t=Z \Sigma_{i} r_{i} / r_{i}^{3}$ and the sums are over all intermediate states. The direct evaluation of (1) by standard means is difficult because the dominant contribution comes from very highly excited states. Accurate calculations have been attempted for the ground state with $Z$ up to 10 (Schwartz 1961; Aashamar and Austvik 1976) and for the low-lying excited states of $\mathrm{He}$ and $\mathrm{Li}^{+}$(Suh and Zaidi 1965, Ermolaev 1975). For the heavier two-electron ions, DeSerio et al (1981) suggest the ad hoc prescription

$$
\ln (\varepsilon(1 \mathrm{~s} n l) / R y)=\ln (\varepsilon(1 \mathrm{~s}) / R y)+n^{-3} \ln (\varepsilon(n l) / R y)
$$

where $\ln \varepsilon(n l)$ is the corresponding one-electron Bethe logarithm and $R y$ is the Rydberg constant. Although (2) appears to give reasonably good agreement with experiment for the $1 \mathrm{~s} 2 \mathrm{~s}^{3} \mathrm{~S}_{1}-1 \mathrm{~s} 2 \mathrm{p}{ }^{3} \mathrm{P}_{0}$ and ${ }^{3} \mathrm{P}_{2}$ transitions, it has no rigorous theoretical foundation.

The purpose of this letter is to report the first $Z^{-1}$ expansion calculation of the Bethe logarithm for two-electron ions. The results for the ground state are compared 
with the direct variational calculations of Aashamar and Austvik (1976). We start by inserting the well known $Z^{-1}$ expansions

$$
\begin{aligned}
& \psi_{n}=\psi_{n}^{0}+Z^{-1} \psi_{n}^{1}+\ldots \\
& E_{n}=Z^{2}\left(E_{n}^{0}+Z^{-1} E_{n}^{1}+\ldots\right)
\end{aligned}
$$

into (1) to obtain

$$
\ln \varepsilon(n L S)=A / B
$$

with

$$
\begin{aligned}
& A=Z^{4}\left[A_{0}+A_{1} Z^{-1}+2(\ln Z)\left(B_{0}+B_{1} Z^{-1}\right)+\ldots\right] \\
& B=Z^{4}\left(B_{0}+B_{1} Z^{-1}+\ldots\right) .
\end{aligned}
$$

Here, $A$ and $B$ are the numerator and denominator of (1) respectively. The expansion of $B$ can easily be obtained from the identity (in atomic units)

$$
B=2 \pi Z\left\langle\delta^{3}\left(\boldsymbol{r}_{1}\right)+\delta^{3}\left(r_{2}\right)\right\rangle .
$$

Thus $B_{0}=4$ and $B_{1}=-2.670587$ for the ground state (Dalgarno and Stewart 1960, Aashamar 1970). Since $A_{0} / B_{0}$ is the Bethe logarithm $\ln \varepsilon(1 \mathrm{~s})=2.984129-\ln 2$ for hydrogen (in atomic units), this determines $A_{0}=9.1639$. Only $A_{1}$ requires significant additional calculation. It is given by

$$
\begin{aligned}
A_{1}=\sum_{m}\left[2\left\langle\psi_{0}^{0}\right|\right. & \boldsymbol{t}\left|\psi_{m}^{0}\right\rangle\left\langle\psi_{m}^{1}|\boldsymbol{t}| \psi_{0}^{0}\right\rangle \ln \Delta E_{m}^{0} / \Delta E_{m}^{0} \\
& +2\left\langle\psi_{0}^{0}|\boldsymbol{t}| \psi_{m}^{0}\right\rangle\left\langle\psi_{m}^{0}|\boldsymbol{t}| \psi_{0}^{1}\right\rangle \ln \Delta E_{m}^{0} / \Delta E_{m}^{0} \\
& \left.+\left|\left\langle\psi_{0}^{0}|\boldsymbol{t}| \psi_{m}^{0}\right\rangle\right|^{2} \Delta E_{m}^{1}\left(1-\ln \Delta E_{m}^{0}\right) /\left(\Delta E_{m}^{0}\right)^{2}\right]
\end{aligned}
$$

where

$$
\begin{aligned}
& \left|\psi_{m}^{1}\right\rangle=\sum_{k \neq m} \frac{\left|\psi_{k}^{0}\right\rangle\left\langle\psi_{k}^{0}\left|r_{12}^{-1}\right| \psi_{m}^{0}\right\rangle}{E_{m}^{0}-E_{k}^{0}} \\
& E_{m}^{1}=\left\langle\psi_{m}^{0}\left|r_{12}^{-1}\right| \psi_{m}^{0}\right\rangle
\end{aligned}
$$

and

$$
\Delta E_{m}^{j}=E_{m}^{j}-E_{0}^{i}
$$

Both singly and doubly excited states contribute to the sums over $m$ and $k$ in (9) and (10). However, since $t$ is a sum of one-electron operators, only s- and p-type one-electron excited states make non-vanishing contributions. We therefore introduce discrete variational one-electron basis sets of the form

$$
\phi_{n}=\sum_{i=1}^{I} \sum_{j=1}^{J} c_{i, j}^{(n)} r^{i-1} \exp \left(-\alpha_{j} r\right) Y_{l}^{m}(\theta, \phi) \quad n=1,2, \ldots, I \times J
$$

for the $\mathrm{s}$ and $\mathrm{p}$ intermediate states. The linear variational coefficients $c_{i, j}^{(n)}$ are determined by the conditions

$$
\begin{aligned}
& \left\langle\phi_{m}|H| \phi_{n}\right\rangle=\varepsilon_{m} \delta_{m, n} \\
& \left\langle\phi_{m} \mid \phi_{n}\right\rangle=\delta_{m, n}
\end{aligned}
$$


and the $\alpha_{j}$ form a geometric series according to

$$
\alpha_{j}=\alpha_{0} \beta^{j-1} \quad j=1,2, \ldots, J .
$$

The infinite sums in (9) and (10) are then replaced by finite sums over two-electron states constructed from antisymmetrised products of the form $\left(\phi_{m}(1) \phi_{n}(2)-\right.$ $\left.\phi_{m}(2) \phi_{n}(1)\right) / \sqrt{ } 2$.

The value of $A_{1}$ obtained from the basis set (13) depends not only on the total size of the basis set, $I \times J$, but also on the values of the non-linear parameters $\alpha_{0}$ and $\beta$. It is therefore important that regions of stability exist with respect to variations in $\alpha_{0}$ and $\beta$, and that $A_{1}$ converges to a definite answer as $I$ or $J$ is increased. We found that these conditions are met only if $J \geqslant 2$, and the $i=1$ term in (13) is retained for the p-type basis states. The latter point follows from a consideration of oscillator strength sum rules (Dalgarno and Epstein 1969). The best convergence and stability were obtained with $J=3$. The multiple exponentials thus introduced into the basis set provide sufficient flexibility to represent adequately both low- and high-energy states. It is then not necessary to treat the two energy regions separately, as done by Schwartz (1961) and Aashamar and Austvik (1976). As a test of the basis set, the hydrogenic Bethe logarithm was calculated by performing directly the summations in (1), with the results shown in table 1 . The extrapolated result agrees with the exact value to within the accuracy of the calculation.

The results for $A_{1}$ are shown in table 2. The tabulated values correspond to a variational minimum with respect to $\alpha_{0}$ and $\beta$. The values vary approximately linearly

Table 1. Test calculation for the one-electron Bethe logarithm using basis sets with $J=3$.

\begin{tabular}{lll}
\hline$I \times J$ & $\ln [\varepsilon(1 \mathrm{~s}) / R y]$ & Error(\%) \\
\hline 6 & 2.9290 & -1.85 \\
9 & 2.9619 & -0.75 \\
12 & 2.9726 & -0.39 \\
15 & 2.9775 & -0.22 \\
18 & 2.9800 & -0.14 \\
Extrapolated & $2.9847 \pm 0.0010$ & $0.02 \pm 0.03$ \\
Exact & $2.984129^{\mathrm{a}}$ & \\
\hline
\end{tabular}

${ }^{a}$ Klarsfeld and Maquet (1973).

Table 2. Values of the expansion coefficient $A_{1}$ for basis sets with $J=3$ and optimised values for $\alpha_{0}$ and $\beta$.

\begin{tabular}{llll}
\hline$I \times J$ & $\alpha_{0}$ & $\beta$ & $A_{1}(\mathrm{au})$ \\
\hline 6 & 0.667 & 5.5 & -5.999 \\
9 & 0.571 & 8 & -6.0972 \\
12 & 0.500 & 10 & -6.1330 \\
15 & 0.444 & 13.5 & -6.14841 \\
18 & 0.400 & 16 & -6.15551 \\
21 & 0.364 & 20 & -6.15966 \\
Extrapolated & & & $-6.169 \pm 0.001$ \\
\hline
\end{tabular}


with $1 / I^{2.5}$ and extrapolate to

$$
A_{1}=-6.169 \pm 0.001 \mathrm{au}
$$

as $I \rightarrow \infty$. Using expansions (6) and (7) in (5), the two-electron Bethe logarithm is then

$$
\begin{aligned}
\ln \frac{\varepsilon\left(1 \mathrm{~s}^{2} \mathrm{~S}\right)}{R y} & =\frac{A_{0}}{B_{0}}+\ln 2+2 \ln Z+\left(\frac{A_{1} B_{0}-A_{0} B_{1}}{B_{0}^{2}}\right) Z^{-1}+\mathrm{O}\left(Z^{-2}\right) \\
& =\ln \left(19.77 Z^{2}\right)-(0.0127 \pm 0.002) Z^{-1}+\mathrm{O}\left(Z^{-2}\right) \\
& \simeq \ln \left[19.77(Z-0.0063)^{2}\right] .
\end{aligned}
$$

Table 3. Comparison of Bethe logarithms for the ground state of helium-like ions.

\begin{tabular}{rllr}
\hline$Z$ & Variational $^{\mathrm{a}}$ & $1 / Z$ expansion $^{\mathrm{b}}$ & \multicolumn{1}{c}{$Z^{2} \Delta$} \\
\hline 2 & $4.37 \pm 0.01$ & 4.364 & $0.02 \pm 0.04$ \\
3 & $5.21 \pm 0.01$ & 5.177 & $0.29 \pm 0.09$ \\
4 & $5.777 \pm 0.003$ & 5.754 & $0.37 \pm 0.05$ \\
5 & $6.214 \pm 0.003$ & 6.201 & $0.33 \pm 0.08$ \\
6 & $6.565 \pm 0.002$ & 6.566 & $-0.02 \pm 0.07$ \\
7 & $6.864 \pm 0.002$ & 6.874 & $-0.50 \pm 0.10$ \\
8 & $7.115 \pm 0.002$ & 7.141 & $-1.70 \pm 0.13$ \\
9 & $7.334 \pm 0.002$ & 7.377 & $-3.50 \pm 0.16$ \\
10 & $7.525 \pm 0.002$ & 7.588 & $-6.31 \pm 0.20$ \\
\hline
\end{tabular}

a Aashamar and Austvik (1976).

${ }^{\mathrm{b}} \ln (\varepsilon / R y)=\ln \left[19.77(Z-0.0063)^{2}\right]$.

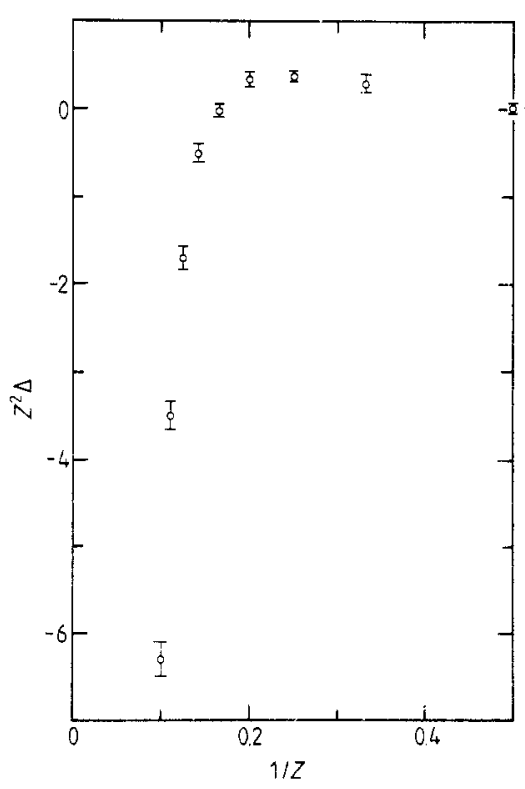

Figure 1. Comparison of results for the two-electron Bethe logarithm as a function of $1 / Z . \Delta$ is the difference between the variational calculation of Aashamar and Austvik (1976) and the value obtained from equation (17). 
The last form expresses the result in terms of a screened nuclear charge. The small magnitude of the screening parameter, 0.0063 , is in harmony with a semi-empirical estimate by Bethe and Salpeter (1957), but the sign is reversed.

The results obtained from (17) are compared in table 3 with the direct variational calculations of the Bethe logarithm by Aashamar and Austvik (1976). The last column gives the difference $\Delta$ between the two sets of results multiplied by $Z^{2}$. Thus $Z^{2} \Delta$ should tend to a constant related to the coefficient of the next term of $\mathrm{O}\left(Z^{-2}\right)$ not included (17). As can be seen from figure 1, the differences for $Z>5$ deviate strongly from the expected behaviour, indicating that the results of Aashamar and Austvik may be less accurate than their estimated error limits. In this high $Z$ region, the $1 / Z$ expansion values calculated from (17) are probably more accurate. Further results for $Z>10$ can be obtained immediately from equation (17).

The use of multiple exponential parameters $\alpha_{j}$ in the basis set (13) plays a crucial role in obtaining stable results with a reasonably small number of terms in the basis set. The technique can be immediately extended to excited states, as will be reported in a future publication.

Research support by the National Science and Engineering Research Council of Canada is gratefully acknowledged. One of us (GWFD) expresses his appreciation to the Ford Motor Company for its hospitality during the preparation of this work.

\section{References}

Aashamar K $1970 \mathrm{Nucl}$. Instrum. Meth. $90263-8$

Aashamar K and Austvik A 1976 Phys. Norv. 8 229-37

Armour I A, Myers E G, Silver J D and Träbert E 1979 Phys. Lett. 75A 45-6

Berry H G, DeSerio R and Livingston A E 1978 Phys. Rev. Lett. 41 1652-5

- 1980 Phys. Rev. A 22998

Bethe H A and Salpeter E E 1957 Quantum Mechanics of One-and Two-Electron Atoms (Berlin: Springer) $\S 74 \gamma$

Buchet J P, Buchet-Poulizac M C, Denis A, Desesquelles J, Druetta M, Grandin J P and Husson X 1981 Phys. Rev. A 23 3354-6

Dalgarno A and Epstein S T 1969 J. Chem. Phys. 50 2837-9

Dalgarno A and Stewart A L 1960 Proc. Phys. Soc. 75441

Davis W A and Marrus R 1977 Phys. Rev. A 15 1963-75

DeSerio R, Berry H G, Brooks R L, Hardis H, Livingston A E and Hinterlong S 1981 Phys. Rev. A 24 $1872-88$

Ermolaev A M 1975 Phys. Rev. Lett. 34 380-3

Holt R A, Rosner S D, Gaily T D and Adam A G 1980 Phys. Rev. A 22 1563-71

Klarsfeld S and Maquet A 1973 Phys. Lett. 43B 201-3

O'Biien R, Silver J D, Jelley N A, Bashkin S, Träbert E and Heckmann P H 1979 J. Phys. B: At. Mol. Phys. 12 L41

Schwartz C 1961 Phys. Rev. 123 1700-5

Stamp M F, Armour I A, Peacock N J and Silver J D 1981 J. Phys. B: At. Mol. Phys. 14 3551-61

Suh K S and Zaidi M H 1965 Proc. R. Soc. A 29 94-105 